Original research article

\title{
Endoscopic transconjunctival surgical approach to intraconal space of the orbit: First clinical experience
}

\author{
Tomasz Lyson $^{a}$, Andrzej Sieskiewicz ${ }^{b}$, Marek Rogowski ${ }^{b}$, \\ Ewa Proniewska-Skretek ${ }^{c}$, Zofia Mariak ${ }^{c}$, Grzegorz Turek ${ }^{a, *}$, \\ Zenon Mariak $^{a}$ \\ a Department of Neurosurgery, Medical University of Bialystok, Bialystok, Poland \\ ${ }^{\mathrm{b}}$ Department of Otolaryngology, Medical University of Bialystok, Bialystok, Poland \\ ${ }^{\mathrm{c}}$ Department of Ophthalmology, Medical University of Bialystok, Bialystok, Poland
}

\section{A R T I C L E I N F O}

\section{Article history:}

Received 16 June 2014

Accepted 15 July 2014

Available online 26 July 2014

Keywords:

Endoscopy

Orbit

Intra-orbital pathology

Surgical approach

Transconjunctival approach

\begin{abstract}
A B S T R A C T
Background and purpose: Recently, a transconjunctival, endoscope-assisted (TEA) approach to the medial intra-orbital space was developed based on cadaver preparations, with an ultimate goal of minimizing disturbances of the anatomic structures of the orbit. However, no report on clinical validation of this promising technique was published thus far. We present our experiences with the TEA approach in two patients.

Material and methods: In emergency conditions, we approached the lateral retrobulbar space of a 42 -year-old male through a $180^{\circ}$ incision close to the corneal limbus; a scrap of metal, which had perforated the globe and resided at its posterior wall, was removed endoscopically. Moreover, we used the TEA approach to remove a tumor from the upper intraconal space in a 63-year-old woman.

Results: In both patients the surgical goal was achieved with no muscle transection and without additional morbidity and complications.

Conclusions: Our experiences with TEA approach suggest that the procedure is clinically feasible, produces no co-morbidity and yields good functional and cosmetic results. As a result, the whole circumference of the retrobulbar space can be conveniently explored.

(C) 2014 Polish Neurological Society. Published by Elsevier Urban \& Partner Sp. z o.o. All
\end{abstract} rights reserved.

\footnotetext{
* Corresponding author at: Department of Neurosurgery, Medical University of Bialystok, Skłodowskiej-Curie 24a Street, 15-276 Bialystok, Poland. Tel.: +48 696454753.

E-mail addresses: lyson_t@vp.pl (T. Lyson), sieska@interia.pl (A. Sieskiewicz), erogowski@onet.eu (M. Rogowski), ewaproniewska@wp.pl (E. Proniewska-Skretek), mariakzo@umb.edu.pl (Z. Mariak), turek.grz@gmail.com, turekgrzegorz@vp.pl (G. Turek), zmariak@umb.edu.pl (Z. Mariak).

http://dx.doi.org/10.1016/j.pjnns.2014.07.008

0028-3843/C 2014 Polish Neurological Society. Published by Elsevier Urban \& Partner Sp. z o.o. All rights reserved.
} 


\section{Introduction}

Surgery of the orbit requires great precision and special care, in order to protect intra-orbital structures and preserve the aesthetics of the face. These goals are now more attainable than ever before due to advances in microsurgical techniques; nevertheless, inherent limitations of microsurgical approaches pertain to the need of bone excision to the extent sufficient for fair illumination of the operating field [1,2]. These factors do not limit endoscopic techniques and, with the advances in extended transnasal approaches, the entire skull base, including the orbit, could be approached endoscopically $[3,4]$.

Transnasal endoscopic approach, through the lamina papyracea exposed with ethmoidectomy and sphenoidectomy, offers a convenient access to the medial and inferior part of the orbit. However, the procedure is technically demanding and, thus, not widespread: the hitherto published clinical material is restricted to case reports only, and very short clinical series focused on tumor biopsies, and decompression in orbital trauma or in endocrine orbitopathy [5-7].

Transnasal endoscopic approach to the medial intraconal space, limited by the optic nerve, medial rectus muscle and the eye bulb, becomes challenging if the target is localized within the anterior part of the orbit; this results from a very awkward transnasal trajectory to this region. Traditionally, the task befalls the ophthalmologist who approaches the pathology with microscopic transconjunctival medial orbitotomy in such situations [1,2,8-10]. However, penetration of this space with standard tools is also limited as the medial rectus muscle has to be detached/transected and an array of critical structures behind the globe has to be pushed and displaced in order to reach the surgical target under microscopic view.

In 2008 Pillai et al. [11] published the results of an anatomical cadaveric study of endoscopic transconjunctival approach to the entire medial intraconal space through a $180^{\circ}$ incision adjacent to the corneal limbus. Advantages of this technique include excellent visualization with neutral or angled optics, limited retraction/dissection of normal tissues, and avoiding detachment of the medial rectus. Surprisingly, no reports on practical application of this promising endoscopic approach to retrobulbar pathologies were published to date. Having a 7-year experience in endoscopic interventions within the orbit, we decided to use this technique in an emergency setting, for removing an intra-orbital metal foreign body, which traversed the eye bulb, and then for excision of an intraconal tumor in another patient. The aim of this study was to present technical aspects and unique possibilities offered by transconjunctival endoscopy-assisted (TEA) approach to the retrobulbar space.

\section{Materials and methods}

An endoscopic team, comprising of a neurosurgeon and otolaryngologist, was asked by the ophthalmologist on call for emergency intervention in a 42-year-old male. The patient was working with a hammer and has been hit by a $4-\mathrm{mm}$ splinter of metal which pierced the inferomedial part of his left
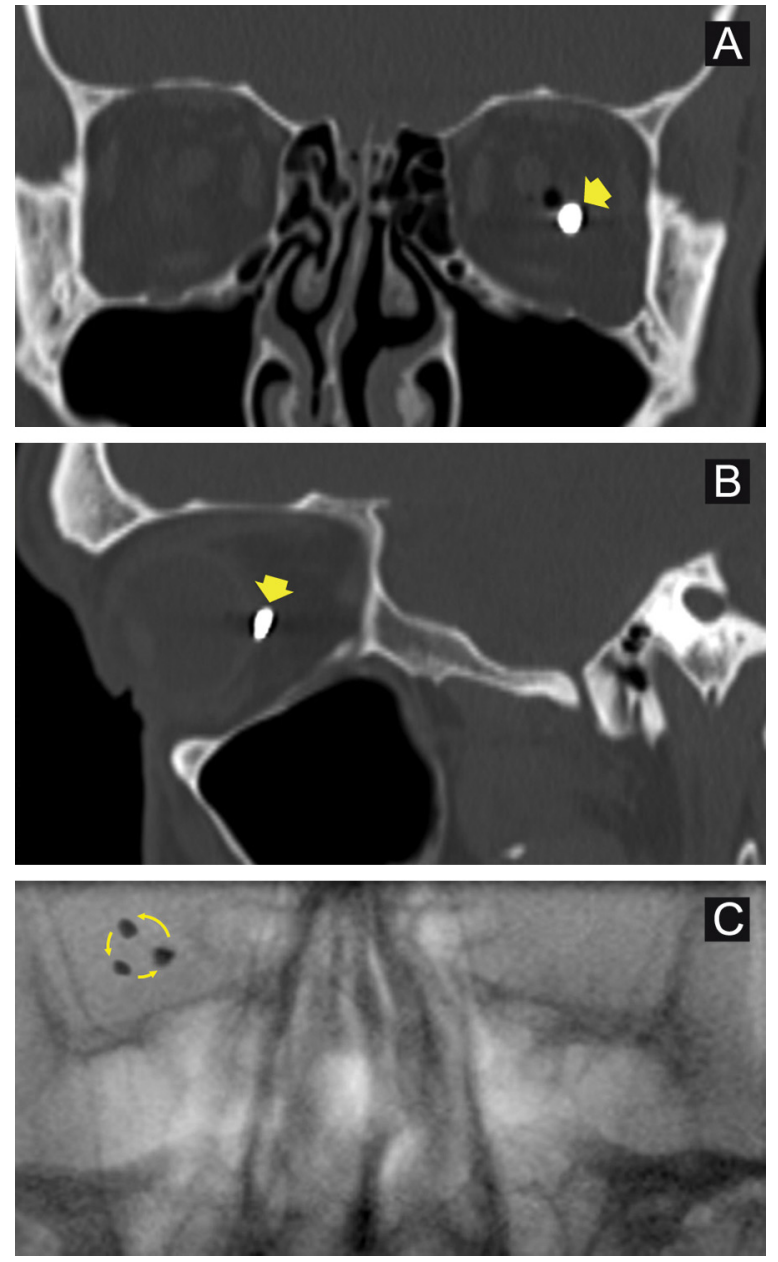

Fig. 1 - Coronal (A) and sagittal (B) CT scans showing a metal scrap (arrow) affixed to the posterior wall of the eye bulb, inferiorly and laterally to the optic nerve. The three superimposed fluoroscopic images (C) demonstrate attachment of the foreign body to the wall of the eye globe: arrows describe movements of the foreign body corresponding to eye movements.

globe, with the inlet $2 \mathrm{~mm}$ from the corneal limbus and the outlet $5 \mathrm{~mm}$ inferolaterally to the optic nerve. His vision was present, but impaired to $5 / 50$, and the motility of the globe was fully preserved. Fundoscopy revealed a small amount of blood in the vitreous body, near the outlet perforation, but the splinter itself was not visible. CT showed the metal scrap extending from the posterior wall of the eye bulb, which moved together with the globe on fluoroscopy (Fig. 1). After critically considering all possible options (magnet extraction, microscopic Krönlein orbitotomy), a decision was made to use the lateral TEA approach to reach the foreign body.

Typical $30^{\circ}$ Hopkins II telescopes $(18 \mathrm{~cm}, \phi 4 \mathrm{~mm}$, Karl Storz Endoscope), attached to an HD camera and connected to a standard xenon light source (Xenon Nova 175), were used. Most of manipulations were performed with free hand technique; only for some maneuvers, the telescope was attached to a holder and fixed. Only anatomical landmarks (lateral rectus muscle, optic nerve and eye bulb surface) could 

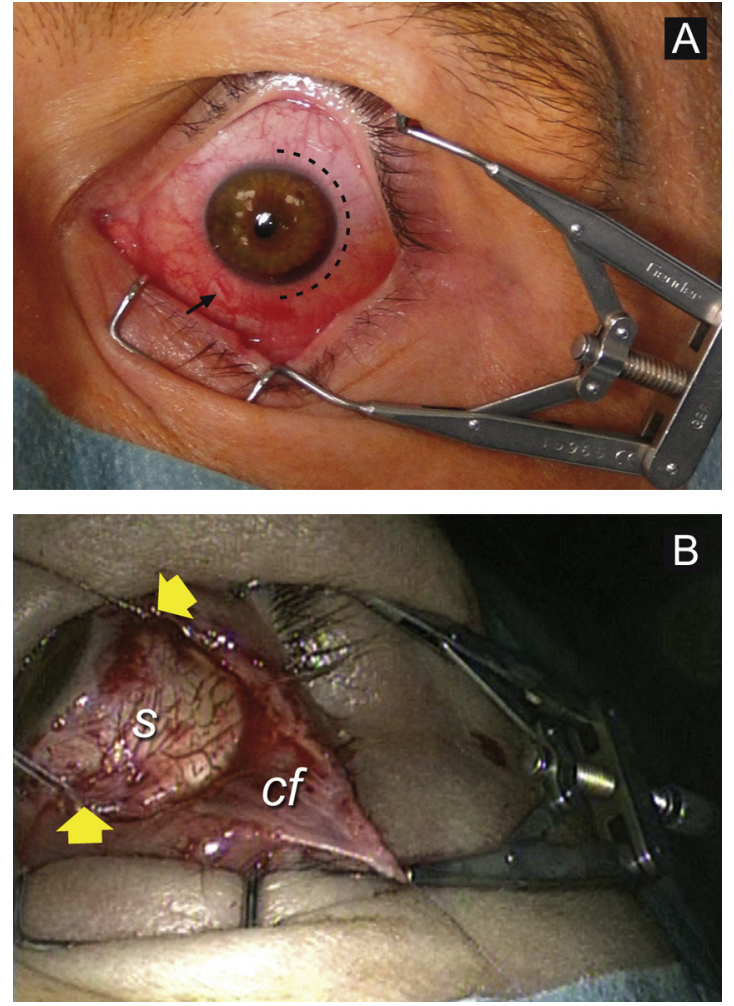

Fig. 2 - (A) Inlet wound of the conjunctiva and sclera (arrow) and conjunctival incision marked with a dotted line. (B) Exposition of the sclera(s), created by gentle rotation of the globe medially with stay sutures stitched to the lateral and inferior rectus muscles (arrows), and by elevation of the conjunctival flap and taking it aside (cf).

be used for surgical guidance in the case of this patient; neuronavigation was ineffective, with the scrap moving with the eye globe.

The eyelids were retracted with a lid speculum and the inlet wound was sutured with two stitches. The procedure begun with $180^{\circ}$ lateral incision close to the corneal limbus, and pulling away the elevated conjunctival Tenon flap as much laterally as possible with the help of stay sutures (Fig. 2). Additional stay sutures were placed on the insertion of the lateral and inferior rectus muscle, and the globe was gently rotated medially. Special care was taken not to compress the pierced globe. The team was prepared to perform lateral orbitotomy with an ultrasound bone cutter if excessive retraction proved necessary. Nevertheless, only pushing the intra-orbital content off the eye bulb with a spatula proved sufficient for creating a corridor up to the region of the optic nerve. A $30^{\circ}$ telescope was introduced obliquely into this narrow retrobulbar space, with the tip of the endoscope pointing into medial direction behind the eye bulb. This was achieved without transection of the lateral rectus muscle. Knowing that the foreign body was stuck somewhere between the optic nerve and the lateral rectus muscle, the tissue was separated gently from the globe along this plane, to eventually visualize the outlet of the optic nerve and the tear (Fig. 3A). A
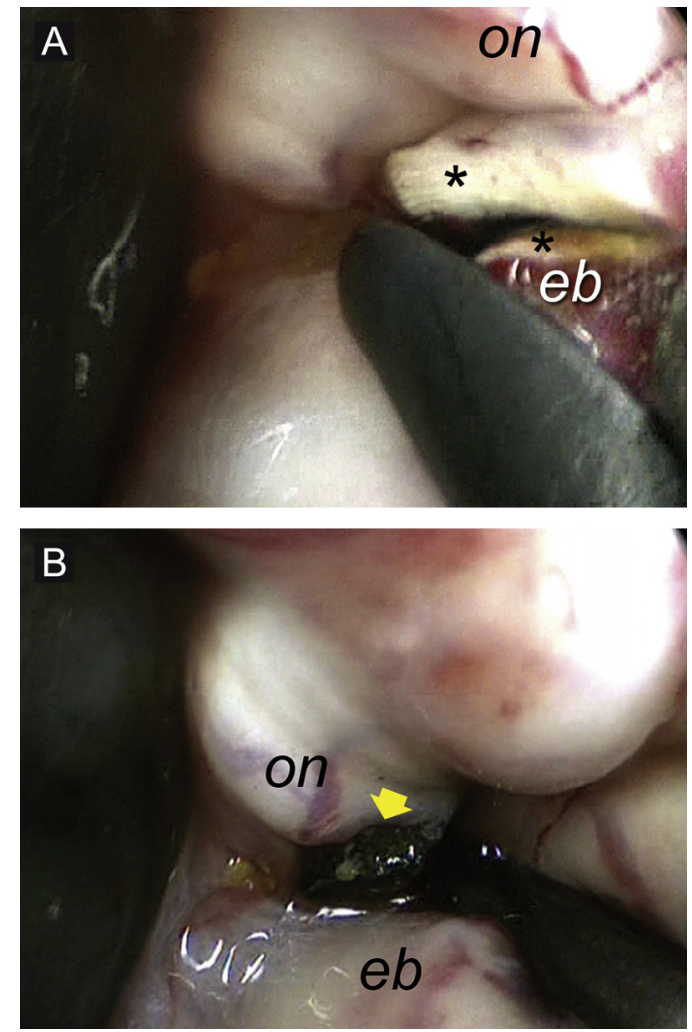

Fig. 3 - (A) Inspection with a $30^{\circ}$ telescope demonstrates the outlet wound within the posterior wall of the orbit, between two asterisks. (B) The foreign body was found to dislodge during preparation and had to be pursued with an additional few $\mathrm{mm}$ preparation along the curvature of the eye bulb (arrow). on - optic nerve, eb - eye bulb.

small magnet was mounted on a clamp and the foreign body was removed under direct vision (Fig. 3B). The tear in the posterior wall of the eye bulb was sealed with a patch of Tachosil ${ }^{\circledR}$ (Nycomed Austria GmbH). The whole procedure was completed within $30 \mathrm{~min}$ by suturing of the conjunctiva.

Another patient, a 63-year-old female, suffered from double vision and left-sided exophthalmos. Her vision acuity was preserved, but motility of the globe was limited. CT and MRI showed an enhancing tumor $(40 \mathrm{~mm} \times 20 \mathrm{~mm} \times 15 \mathrm{~mm}$ ) localized in the upper supra- and retrobulbar space of the left orbit, adjacent to the globe (Fig. 4). Seemingly "easy" biopsy of the tumor proved unsuccessful on two attempts and therefore the patient was referred by the ophthalmological department to a neurosurgeon, with a plan to approach the lesion for biopsy with a more extensive transconjunctival incision and under a direct endoscopic guidance.

The procedure was performed under neuronavigation (Stealth Station Treon Plus, Medtronic, United States). The endoscopic instrumentation for this procedure was essentially the same as described above, but a $0^{\circ}$ telescope was used instead of a $30^{\circ}$ optic. After retraction of the eyelid, the TEA approach begun with a $180^{\circ}$ incision of the conjunctiva along the superomedial corneal limbus. The conjunctival Tenon flap was elevated and pulled up with stay stitches to expose the 

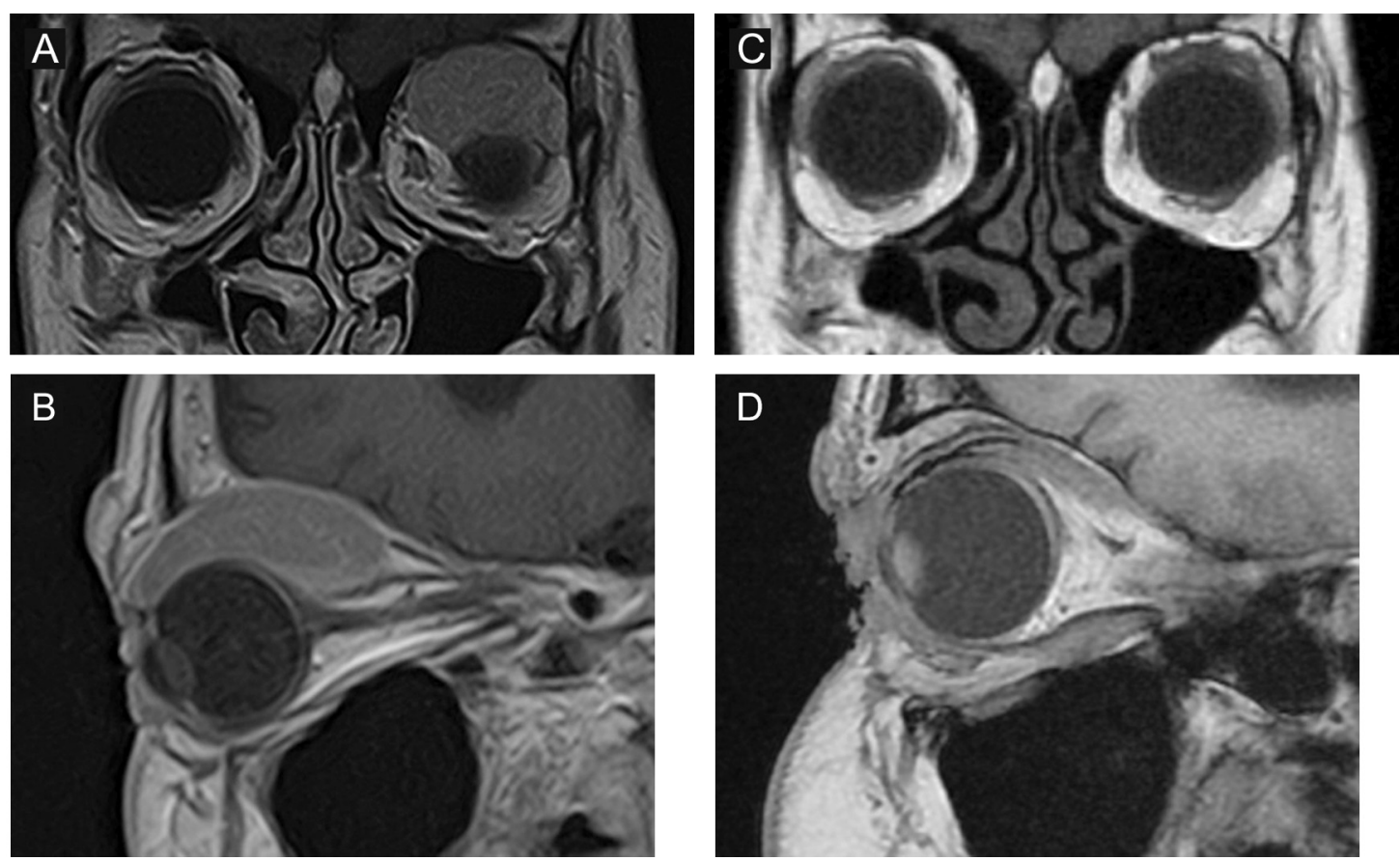

Fig. 4 - Coronal (A) and sagittal (B) T1-weighted post-gadolinium MR scans showing the enhancing tumor $(40 \mathrm{~mm} \times 20 \mathrm{~mm} \times 15 \mathrm{~mm}$ ) in the upper supra- and retrobulbar space of the left orbit, adjacent to the globe. (C, D) Postoperative coronal and sagittal scans showing gross total excision of the tumor with preservation of the superior rectus muscle.

capsule of the tumor. This allowed to create a narrow space behind the eye bulb; after slipping in the telescope, the space was gently advanced backward, along the inner interface of the tumor, using blunt preparation. The upper rectus muscle was identified and separated from the tumor. Perfect visualization of the dissected tissues encouraged the operating team to pursue preparation along the whole circumference of the tumor capsule, all the time avoiding compression of the globe, as well as any damage of the intra-orbital fat, muscles and vascular structures. Total resection of the lesion, which eventually was diagnosed as pseudotumor, was achieved. The procedure took $40 \mathrm{~min}$ and was completed with suturing of the conjunctiva.

\section{Results}

The foreign body in the first patient was successfully removed with no need for transection of intra-orbital muscles; due to the use of angled optics and malleable tools we avoided excessive compression of the perforated eyeball. Furthermore, the use of endoscopic technique enabled us to achieve another important objective, i.e. sealing of the outlet wound with a patch of Tachosil. No new symptoms or functional deficits were observed postoperatively, and we did not note any deterioration of visual function. The vision was maintained at the initial level of 5/50, and the motility of the globe was fully preserved. Postoperative fundoscopy confirmed the presence of small amount of blood in the vitreous body, near the outlet perforation. Although we still consider vitrectomy, the procedure was postponed due to potential risk of Tachosil patch detachment; the patch, which seals the wound at the posterior aspect of the eye globe, could be detached due to an intraoperative increase in intraocular pressure during vitrectomy. Nevertheless, the cosmetic result of the surgery is good; although the conjunctiva was still somewhat irritated at 1 month after the procedure, the conjunctival scar was no longer discernible.

The biopsy, initially attempted in the second patient, eventually turned out to be a total removal of the tumor with preserved integrity and function of the orbital muscles and nerves. Double vision and left-sided exophthalmos resolved along with a decrease in postoperative edema. Vision acuity was fully preserved postoperatively and the motility of the globe resumed gradually. The cosmetic result, estimated a year after the procedure, was assessed as good as no visible conjunctival scar and only mild left-sided ptosis were documented.

Histopathologic examination confirmed an inflammatory character of the lesion, and no signs of tumor recurrence were documented on MRI performed a year after the procedure.

\section{Discussion}

Our experiences with the two hereby presented patients show that endoscopic transconjunctival approach to surgical management of intra-orbital retrobulbar pathologies is feasible and safe. However, it should be remembered that the problem might have been also resolved with traditional external lateral and superior transcranial orbitotomy in both cases [1,2,8-10]. Nevertheless, both patients were dealt with in a minimally 
invasive way due to TEA approach, avoiding external skin incision/scarring and bone removal, which is known to increase the risk of enophthalmos. Another viable alternative could have been transconjunctival microsurgical approach, especially in the patient with orbital tumor. Compared to the latter technique, the main advantages of endoscopy include preservation of extraocular muscle integrity and function, as well as of minimizing the eye globe compression.

According to Pillai et al. [11], the endoscope-assisted direct approach to the retrobulbar space is mainly meant as a pathway to the medial intraconal space, predominantly for optic nerve decompression. We were forced to use this approach for lesions situated in the inferolateral and superior part of the orbit. Our hereby presented experiences suggest that the endoscopic exploration can be pursued in any direction along the curvature of the eye globe, in the retrobulbar space. During his/her maneuvers, the surgeon can respect the curvature of the eye globe much better than with a microscope (which, obviously, gives no possibility of looking "around the corner"); this results from use of angled optics and tilted/malleable tools. This virtue of the endoscopic technique proved crucial in the patient with perforated globe.

If the surgical target is adjacent to the posterior aspect of the globe, the transconjunctival endoscopic approach seems most direct and least invasive option. First of all, contrary to microsurgical technique, endoscopic exploration usually can be successfully executed avoiding extraocular muscle transection [11]. This minimizes the risk of postoperative ocular misalignment. Furthermore, relaxing incisions of the conjunctiva, sometimes necessary with microscopic approach, proved unnecessary in both our patients. Our findings are consistent with cadaveric preparation-based observations of Pillai et al. [11], according to whom endoscopic assist is beneficial in the preservation of the tiny capsule and septa that surround and stabilize the orbital fat. Leaving the fat undisturbed as much as possible is essential for both the comfort of surgery and its cosmetic and functional outcome. Importantly, we managed to pursue preparation along the anatomic planes and avoided fat prolapse in both our patients.

The idea of transconjunctival endoscopic approach to the orbit was originally based solely on a cadaveric study, and its clinical application has not been reported to date. Also our experience with the use of endoscopy in such a close vicinity to the eye globe and the optic nerve is very limited. Therefore, one should consider potential limitations of the method, among them still vastly unknown effects of excessive illumination and dissemination of heat from the tip of the endoscope. A recent study of thermal effects of endoscopy during skull base surgery documented an unexpectedly high local temperature excursions in these anatomically closed spaces [12]. Although these effects have not been studied during intra-orbital surgery, one must be cautious when working with an endoscope within artificially created narrow spaces along the surface of the eye globe and in close proximity to the optic nerve.

Although the data on the consequences of intensive exposition to light are limited, such effects could also appear with endoscopic maneuvers in a close vicinity to the eye and optic nerve. Therefore, great care must be exerted in order to protect the patient against potentially harmful effects of excessive light and temperature: any direct illumination of the globe, especially the iris, should be reduced by covering the vital structures with pads, frequent flushing of the operative field and, in particular, avoiding prolonged work under the telescope, which has been fixed in a holder. Finally, the endoscopic team must gain a certain level of technical proficiency before entering these regions of the orbit, to make the procedure as short as possible.

\section{Conclusions}

Our initial experiences with TEA approach suggest that the procedure is clinically feasible, produces no co-morbidity and yields good functional and cosmetic results. The whole circumference of the retrobulbar space can be conveniently explored with this approach. High proficiency of the surgical team and special care are indispensable, because the operating time must be reduced to a minimum, and the eye and the optic nerve must be incessantly protected against potentially harmful effects of heat and excessive illumination.

\section{Conflict of interest}

None declared.

\section{Acknowledgement and financial support}

None declared.

\section{Ethics}

The work described in this article has been carried out in accordance with the Code of Ethics of the World Medical Association (Declaration of Helsinki) for experiments involving humans; uniform requirements for manuscripts submitted to biomedical journals.

\section{R EFERE N C E S}

[1] Khan AM, Varvares MA. Traditional approaches to the orbit. Otolaryngol Clin North Am 2006;39:895-909.

[2] Koerbel A, Ferreira VR, Kiss A. Combined transconjunctivaleyebrow approach providing minimally invasive access to all orbital quadrants. Technical note. Neurosurg Focus 2007;23:E10.

[3] Kassam AB, Prevedello DM, Carrau RL, Snyderman CH, Thomas A, Gardner P, et al. Endoscopic endonasal skull base surgery: analysis of complications in the authors' initial 800 patients. J Neurosurg 2011;114: 1544-68.

[4] Paluzzi A, Gardner P, Fernandez-Miranda JC, Snyderman C. The expanding role of endoscopic skull base surgery. Br J Neurosurg 2012;26(5):649-61. 
[5] Murray KP, Mirani NM, Langer PD, Liu JK, Eloy JA. Endoscopic transnasal septotomy for contralateral orbital apex venous angioma resection and decompression. Orbit 2013;32:36-8.

[6] Sia DI, Chan WO, Wormald PJ, Davis G, Selva D. Decompression of benign orbital apex lesion via medial endoscopic approach. Orbit 2012;31:344-6.

[7] Sieskiewicz A, Lyson T, Mariak Z, Rogowski M. Endoscopic trans-nasal approach for biopsy of orbital tumours using image-guided neuro-navigation system. Acta Neurochir (Wien) 2008;150:441-5.

[8] Gdal-On M, Gelfand YA. Surgical outcome of transconjunctival cryosurgical extraction of orbital cavernous hemangioma. Ophthalmic Surg Lasers 1998;29:969-73.
[9] Gurkanlar D, Gonul E. Medial microsurgical approach to the orbit: an anatomic study. Minim Invasive Neurosurg 2006;49:104-9.

[10] Maroon JC, Kennerdell JS. Surgical approaches to the orbit. Indications and techniques. J Neurosurg 1984;60:1226-35.

[11] Pillai P, Lubow M, Ortega A, Ammirati M. Endoscopic transconjunctival surgical approach to the optic nerve and medial intraconal space: a cadaver study. Neurosurgery 2008;4(Suppl. 2):204-8. discussion 208-9.

[12] Lyson T, Sieskiewicz A, Sobolewski A, Rutkowski R, Kochanowicz J, Turek G, et al. Operative field temperature during transnasal endoscopic cranial base procedures. Acta Neurochir (Wien) 2013;155:903-8. 